\title{
Bayesian Test for Lifetime Performance Index of Exponential Distribution under Symmetric Entropy Loss Function
}

\author{
Guobing Fan \\ School of Mathematics and Statistics, Hunan University of Finance and Economics, Changsha, China \\ Email address: \\ fanguobingmath@163.com \\ To cite this article: \\ Guobing Fan. Bayesian Test for Lifetime Performance Index of Exponential Distribution under Symmetric Entropy Loss Function. \\ Mathematics Letters. Vol. 4, No. 1, 2018, pp. 20-24. doi: 10.11648/j.ml.20180401.15
}

Received: March 1, 2018; Accepted: March 27, 2018; Published: May 4, 2018

\begin{abstract}
The task of this paper is to estimate the lifetime performance index of Exponential distribution. A Bayesian test procedure is established under symmetric entropy loss function. Firstly, Bayesian estimation of life performance index is obtained, then a Bayesian test procedure for lifetime performance index is proposed. Finally, an applied example is used to illustrate the effectiveness of the proposed test method.
\end{abstract}

Keywords: Lifetime Performance Index, Bayesian Test, Exponential Distribution, Symmetric Entropy Loss Function

\section{Introduction}

Process capability indices (PCIs) have been introduced for measuring process reproduction capability of a manufacturing industry [1]. They are important in the manufacturing industry to measure process potential and performance [2]. There are many well-known PCIs have been put forward. For example, the first process capability index $C_{P}$ is first introduced by Juran in reference [3]. Then other PCIs: $C_{p k}, C_{p m}, C_{p m k}$ are proposed respectively [4-6]. The study and applications of PCIs have drawn great attention. For example, Abdolshah et al. [7] studied the application of Cpmk in the fuzzy context and compare it with other fuzzy PCIs. Kurniati [8] proposed a variables resubmitted sampling scheme for one-sided specification limit based on one-sided PCIs. Seifi and Nezhad [9] developed a variable sampling plan for resubmitted lots based on PCI and Bayesian approach.

To assess the products with the larger-the-better type characteristics, Montgomery [10] proposed a special unilateral specification PCI, named as lifetime performance index $C_{L}$, which is defined as follows

$$
C_{L}=\frac{\mu-L}{\sigma}
$$

where $L$ is the lower bound of the specifications.
The statistical inference of lifetime performance index for products whose lifetime distributed different distributions have been widely studied in recent years. For example, Lee et al. [11] based on type II right censored sample discussed the computational procedure of performance assessment of lifetime index of normal products in the fuzzy context. Liu and Ren [12] discussed the Bayesian point estimation and test of lifetime performance index when the products' lifetime is distributed with exponential distribution under progressively type II censored samples. Wu et al. [13] designed acceptance-sampling plans for an exponential population based on a lifetime-performance index by minimize the number of failures required during inspection. Soliman [14] discussed the Bayesian and non-Bayesian estimators for lifetime performance index based on progressive first-failure censoring samples arising from the exponentiated Frechet distribution. Li [15] discussed the Bayesian estimation and test procedure of lifetime performance index for Ailamujia distribution under squared error loss function.

Suppose that $X$ is the products' lifetime, and it distributed with exponential distribution whose probability density function (pdf) and cumulative distribution function (cdf) respectively:

$$
f(x \mid \theta)=\theta e^{-\theta x}, \quad x>0
$$




$$
F(x ; \theta)=1-\theta e^{-\theta x}, \quad x>0
$$

Here, $\theta>0$ is the unknown parameter.

The aim of this paper is to study the Bayesian statistical inference of lifetime performance index $C_{L}$. The Bayesian estimation and the Bayesian test procedure of $C_{L}$ will be derived. The organization of this article is as follows. Section 2 will recall some properties of the lifetime performance index of product with the exponential distribution. The relationship between $C_{L}$ and conforming rate will be also discussed. Furthermore, the Bayesian estimator of $C_{L}$ based on the conjugate Gamma prior distribution is also obtained under symmetric entropy loss function in Section 3. A new Bayesian hypothesis testing procedure of lifetime performance index is developed in Section 4, and an application example is given in Section 5. Finally, conclusions are given in Section 6.

\section{Lifetime Performance Index}

Suppose that $X$ is the lifetime of such a product whose lifetime distribution is exponential distribution with pdf (2). Then the process mean $\mu=E X=1 / \theta$ and the process standard deviation $\sigma=\sqrt{\operatorname{Var}(\mathrm{X})}=1 / \theta$. Then the parameter $\theta^{-1}$ is often called the mean time. Then the lifetime performance index $C_{L}$ of exponential product can be rewritten as follows

$$
\mathrm{C}_{\mathrm{L}}=\frac{\mu-\mathrm{L}}{\sigma}=\frac{1 / \theta-\mathrm{L}}{1 / \theta}=1-\theta \mathrm{L}
$$

The failure rate function $r(x)$ can be derived as

$$
\mathrm{r}(\mathrm{x})=\frac{\mathrm{f}(\mathrm{x} \mid \theta)}{1-\mathrm{F}(\mathrm{x} \mid \theta)}=\frac{\theta \exp (-\theta \mathrm{x})}{\exp (-\theta \mathrm{x})}=\theta
$$

From (4) and (5), we can see that the larger the mean $1 / \theta$ is, the smaller the failure rate and larger the lifetime performance index $\mathrm{C}_{\mathrm{L}}$ are. Therefore, the lifetime performance index $\mathrm{C}_{\mathrm{L}}$ can reasonably and accurately represent the product performance of new products.

Moreover, if the lifetime $X$ of a product exceeds the lower specification limit $\mathrm{L}$, then the product is defined as a conforming rate, and can be defined as

$$
\begin{gathered}
P_{r}=P(X \geq L)=\int_{L}^{\infty} \theta \exp (-\theta x) d x \\
=e^{-\theta L}=e^{C_{L}-1},-\infty<C_{L}<1
\end{gathered}
$$

According to the equation (6), we can see there exists a strictly increasing relationship between conforming rate $\mathrm{P}_{\mathrm{r}}$ and the lifetime performance index $\mathrm{C}_{\mathrm{L}}$. Table 1 lists values of $\mathrm{C}_{\mathrm{L}}$ and the corresponding conforming rate $\mathrm{P}_{\mathrm{r}}$. When the values of $\mathrm{C}_{\mathrm{L}}$ which are not listed in Table 1, the conforming rate $P_{r}$ can be derived through interpolation.

Table 1. The lifetime performance index $C_{L}$ versus the conforming rate $P_{r}$.

\begin{tabular}{llllll}
\hline $\mathbf{C}_{\mathbf{l}}$ & $\mathbf{P}_{\mathbf{r}}$ & $\mathbf{C}_{\mathbf{l}}$ & $\mathbf{P}_{\mathbf{r}}$ & $\mathbf{C}_{\mathbf{l}}$ & $\mathbf{P}_{\mathbf{r}}$ \\
\hline$-\infty$ & 0.00000 & 0.125 & 0.41686 & 0.575 & 0.65377 \\
-2.75 & 0.02352 & 0.175 & 0.43823 & 0.625 & 0.68729 \\
-2.50 & 0.03020 & 0.200 & 0.44933 & 0.650 & 0.70469 \\
-2.00 & 0.04979 & 0.250 & 0.47237 & 0.700 & 0.74082 \\
-1.50 & 0.08209 & 0.300 & 0.49659 & 0.750 & 0.77880 \\
-1.25 & 0.10540 & 0.325 & 0.50916 & 0.775 & 0.79852 \\
-1.00 & 0.13534 & 0.350 & 0.52205 & 0.800 & 0.81873 \\
-0.75 & 0.17377 & 0.375 & 0.53526 & 0.825 & 0.83946 \\
-0.50 & 0.22313 & 0.400 & 0.54881 & 0.850 & 0.86071 \\
-0.25 & 0.28650 & 0.425 & 0.56270 & 0.875 & 0.88250 \\
0.000 & 0.36788 & 0.450 & 0.57695 & 0.900 & 0.90484 \\
0.025 & 0.37719 & 0.475 & 0.59156 & 0.925 & 0.92774 \\
0.050 & 0.38674 & 0.500 & 0.60653 & 0.950 & 0.95123 \\
0.075 & 0.39657 & 0.525 & 0.62189 & 0.975 & 0.97531 \\
\hline
\end{tabular}

The conforming rate can be calculated by dividing the number of conforming products by total number of products sampled. To accurately estimating rate, Montgomery [10] suggested that the sample size must be enough large. However, a large number of samples are usually not practical from the point of cost. In addition, a large sample is also not practical due to time limitation or other restrictions such as material resources, mechanical or experimental difficulties and so on. Since a one-to-one mapping exists between the conforming rate $P_{r}$ and the lifetime performance index $C_{L}$. Therefore, lifetime performance index can be not only a flexible and effective tool to evaluate the products' performance, but also be a effective tool to estimate the conforming rate $\mathrm{P}_{\mathrm{r}}$.

\section{Estimation}

\subsection{Maximum Likelihood Estimation}

Let $X_{1}, X_{2}, \cdots, X_{n}$ be a lifetime of sample from the exponential distribution with $\operatorname{pdf}(2), x=\left(x_{1}, x_{2}, \cdots, x_{n}\right)$ is the observation of $X=\left(X_{1}, X_{2}, \cdots, X_{n}\right)$ and $t=\sum_{i=1}^{n} x_{i}$ is the observation of statistic $T=\sum_{i=1}^{n} X_{i}$. Then the likelihood function corresponding to $\operatorname{pdf}(2)$ is given by

$$
\mathrm{L}(\mathrm{x} ; \theta)=\prod_{\mathrm{i}=1}^{\mathrm{n}} \mathrm{f}(\mathrm{x} \mid \theta)=\prod_{\mathrm{i}=1}^{\mathrm{n}} \theta \exp \left(-\theta \mathrm{x}_{\mathrm{i}}\right)=\theta^{\mathrm{n}} \mathrm{e}^{-\theta t}
$$

The maximum likelihood estimator of $\theta$ can be easily derived from the log-likelihood equation $\frac{d \operatorname{Ln}[l(\theta ; x)]}{d \theta}=0$ as follows:

$$
\hat{\theta}_{M}=\frac{n}{T}
$$

We can also easily prove that $T$ is a random variable distributed with Gamma distribution $\Gamma(n, \theta)$ with the 
following probability density function:

$$
f_{T}(t ; \theta)=\frac{\theta^{n}}{\Gamma(n)} t^{n-1} e^{-\theta t}, \quad t>0, \theta>0
$$

\subsection{Bayes Estimation}

This section will discuss the Bayesianian estimation of the lifetime performance index of exponential distribution with pdf (2) under symmetric entropy loss function (Zhao [16] and $\operatorname{Li}[17])$

$$
L(\hat{\theta}, \theta)=\frac{\hat{\theta}}{\theta}+\frac{\theta}{\hat{\theta}}-2
$$

We are interested in estimating $\theta$ under the symmetric entropy loss function (10).

Lemma 1 Let $X_{1}, X_{2}, \cdots, X_{n}$ be a lifetime of sample from the exponential distribution with $\operatorname{pdf}(2), x=\left(x_{1}, x_{2}, \cdots, x_{n}\right)$ is the observation of $X=\left(X_{1}, X_{2}, \cdots, X_{n}\right)$, Then under the symmetric entropy loss function (10), the unique Bayesian estimator of $\theta$, say $\hat{\theta}_{B}$, is given by

$$
\hat{\theta}_{B}=\left[\frac{E(\theta \mid X)}{E\left(\theta^{-1} \mid X\right)}\right]^{1 / 2}
$$

Proof. Obviously, the symmetric loss in (11) is strictly convex, then the unique Bayesian estimator is obtained from the relation

$$
\frac{d E[L(\hat{\theta}, \theta) \mid X]}{d \theta}=0
$$

which reduces to (11).

Assume that the prior distribution of the parameter $\theta$ is the Gamma prior distribution $\Gamma(\alpha, \beta)$, with pdf

$$
\pi(\theta ; \alpha, \beta)=\frac{\beta^{\alpha}}{\Gamma(\alpha)} \theta^{\alpha-1} e^{-\beta \theta}, \quad \alpha, \beta>0, \theta>0
$$

Then combining the likelihood function (7) with the prior pdf (12), the posterior pdf of $\theta$ can be derived using Bayes' Theorem as follows

$$
\begin{aligned}
h(\theta \mid x) & \propto l(\theta ; x) \cdot \pi(\theta) \\
& \propto \theta^{n} e^{-\theta t} \cdot \frac{\beta^{\alpha}}{\Gamma(\alpha)} \theta^{\alpha-1} e^{-\beta \theta} \\
& \propto \theta^{n+\alpha-1} e^{-(\beta+t) \theta}
\end{aligned}
$$

That is

$$
\theta \mid X \sim \Gamma(n+\alpha, \beta+T) .
$$

Then under the symmetric entropy loss function (10), the Bayesian estimator of $\theta$ can be derived as follows

$$
\begin{aligned}
& \hat{\theta}_{B}=\left[\frac{E(\theta \mid X)}{E\left(\theta^{-1} \mid X\right)}\right]^{1 / 2}=\left[\frac{\frac{n+\alpha}{\beta+T}}{\frac{\beta+T}{n+\alpha-1}}\right]^{1 / 2} \\
& =\left[\frac{1}{\sqrt{(n+\alpha)(n+\alpha-1)}}(\beta+T)\right]^{-1}
\end{aligned}
$$

Further the Bayesian estimator of $C_{L}$ is

$$
\begin{aligned}
\hat{C}_{B} & =1-\hat{\theta}_{B} L \\
& =1-\left[\frac{\beta+T}{\sqrt{(n+\alpha)(n+\alpha-1)}} \cdot L\right]^{-1}
\end{aligned}
$$

\section{Bayesian Test of Lifetime Performance Index}

This section will propose a Bayesian testing procedure to assess whether the lifetime performance index adheres to the required level. Assume that the required index value of lifetime performance is larger than $c$, where $c$ is the target value. First, we construct the following hypothesis:

$$
H_{0}: C_{L} \leq c \leftrightarrow H_{1}: C_{L}>c .
$$

Let $Y=2 \theta(\beta+T) \mid X$, the for given Significance level $\gamma$, we can easily shown that $\theta \mid X \sim \Gamma(2 n+\alpha, \beta+T)$. Let $\chi_{1-\gamma}^{2}(2(n+\alpha))$ be the $1-\gamma$ percentile of $\chi^{2}(2(n+\alpha))$.

Then

$$
P\left(2 \theta(\beta+T) \leq \chi_{1-\gamma}^{2}(2(n+\alpha)) \mid X\right)=1-\gamma
$$

That is

$$
\begin{gathered}
P\left(\theta \leq \frac{\chi_{1-\gamma}^{2}(2(n+\alpha))}{2(\beta+T)} \mid X\right)=1-\gamma \\
P\left(1-\theta L \geq 1-L \cdot \frac{\chi_{1-\gamma}^{2}(2(n+\alpha))}{2(\beta+T)} \mid X\right)=1-\gamma
\end{gathered}
$$

Then the lower confidence limit of lifetime performance index $C_{L}$ with level $1-\gamma$ can be obtained, as follows:

$$
\underline{L B}=1-\left(1-\hat{C}_{B}\right) \cdot \frac{\chi_{1-\gamma}^{2}(2(n+\alpha))}{2(n+\alpha-1)}
$$

Then the new proposed Bayesian testing procedure of $C_{L}$ is as follows:

(i) For given sample size n, determine the lower lifetime specification limit $L$.

(ii) Under symmetric entropy loss function, calculate the Bayesian estimator 


$$
\hat{\theta}_{B}=\left[\frac{1}{\sqrt{(n+\alpha)(n+\alpha-1)}}(\beta+T)\right]^{-1},
$$

Where $T=\sum_{i=1}^{n} X_{i}$.

(iii) Calculate the $100(1-\gamma) \%$ one-side confidence interval $[\underline{L B}, \infty)$ for lifetime performance index $\mathrm{C}_{\mathrm{L}}$, where $\underline{L B}$ is defined as equation (21).

(iv) The decision rules of statistical test are provided as follows:

If the performance index value $c \notin[\underline{\mathrm{LB}}, \infty)$, we reject to the null hypothesis $H_{0}$ and conclud that the lifetime performance index of product meets the required level.

If the performance index value $c \in[\underline{\mathrm{LB}}, \infty)$,, we accept the null hypothesis $H_{0}$ and conclude that the lifetime performance index does not meet the required level.

\section{Discussion}

To illustrate the feasibility and practicability of the proposed Bayesian testing method, a practical example proposed in reference Nelson [18] (1982, P. 105. Table 1.1) is adopted. There is a life testing experiment in which specimens of a type of electrical insulating fluid were subject to a constant voltage stress. The data set is reported in Table 2.

Table 2. Life testing experiment data.

\begin{tabular}{llllllll}
\hline \multicolumn{2}{l}{ Data Set $(\mathbf{n}=\mathbf{1 9})$} & & & & \\
\hline 0.19 & 0.78 & 1.31 & 2.78 & 0.96 & 4.15 & 12.06 & 6.50 \\
31.75 & 3.16 & 4.85 & 72.89 & 32.52 & 4.67 & 7.35 & 8.27 \\
8.01 & 33.91 & 36.71 & & & & & \\
\hline
\end{tabular}

Balakrishnan et al. [19] analyzed these $n=19$ observations, and they proved that the data set has an exponential distribution with pdf as (2) by using least squares method and goodness of fit test. Gail and Gastwirth [20] also proved the data set can be modeled by exponential model based on Gini statistics. In the follow, we will give the detail steps of the proposed testing procedure about $C_{L}$ as:

(i) Determine the lower lifetime limit $\mathrm{L}=1.04$. That is to say if the lifetime of an electrical insulating fluid exceeds L hours, then the electrical insulating fluid is a conforming product. In order to satisfy the regarding operational performance. The conforming rate $\mathrm{P}_{\mathrm{r}}$ is required to exceed 80 percent. Referring to Table 1 , the value of $C_{L}$ is required to exceed 0.80 . Thus, the performance index target value is $\mathrm{c}=0.80$.

(ii) Establish testing hypothesis as below

$$
H_{0}: C_{L} \leq 0.80 \leftrightarrow H_{1}: C_{L}>0.80 .
$$

(iii) Specified a significance level $\gamma=0.05$.

(iv) Calculate the $100(1-\gamma) \%$ one-side confidence interval $[\underline{L B}, \infty)$ for $\mathrm{C}_{\mathrm{L}}$, where

$$
\underline{L B}=1-\left(1-\hat{C}_{B}\right) \cdot \frac{\chi_{1-\gamma}^{2}(2(n+\alpha))}{2(n+\alpha-1)}
$$

Here we suppose the prior distribution of $\theta$ is the noninformative prior, i.e. $\alpha=\beta=0$. Then the $95 \%$ onesided confidence interval for $C_{L}$ is $[\underline{\mathrm{LB}}, \infty)=[0.8983, \infty)$.

(v) Because of the performance index value $c=0.80 \notin[\underline{\mathrm{LB}}, \infty)$, we reject the null hypothesis $H_{0}: C_{L} \leq 0.80$. Thus, we can conclude that the lifetime performance index of products meets the required level.

\section{Conclusions}

Process capability indices are widely employed in manufacture industry to assess the performance and potential of their process. Lifetime performance index is one of the most important PCIs. This paper studies the Bayesian estimation and Bayesian test of life performance index when the lifetime of products is distributed with exponential distribution. The proposed testing method is easy to complete with the help of some software, such as excel, matlab. The new test procedure can provide reference for the enterprise engineers to assess whether the true performance of products meets the requirements.

\section{Acknowledgements}

This study is partially supported by Social Science Foundation of Hunan Province (No. 15YBA065). The author is grateful to the reviewers for a very careful reading of the manuscript and the suggestions.

\section{References}

[1] Shu M H, Wu H C. Manufacturing process performance evaluation for fuzzy data based on loss-based capability index [J]. Soft Computing, 2012, 16 (1):89-99.

[2] Chen C C, Lai C M, Nien H Y. Measuring process capability index Cpm with fuzzy data [J]. Quality \& Quantity, 2010, 44 (3):529-535.

[3] Juran J M, Gryna F M, Bingham R S J. Quality Control Handbook. New York: McGraw-Hill., 1974.

[4] Eslamipoor R, Hosseini-Nasab H. A modified process capability index using loss function concept [J]. Quality \& Reliability Engineering International, 2016, 32 (2):435-442.

[5] Chen K S, Huang C F, Chang T C. A mathematical programming model for constructing the confidence interval of process capability index $\mathrm{Cpm}$ in evaluating process performance: an example of five-way pipe [J]. Journal of the Chinese Institute of Engineers, 2017, 40 (2):126-133.

[6] Gu X, Ma Y, Liu J, et al. Robust parameter design for multivariate quality characteristics based on process capability index with individual observations [J]. Systems Engineering \& Electronics, 2017, 39 (2):362-368. 
[7] Abdolshah M, Yusuff R M, Hong T S, et al. Measuring process capability index Cpmk, with fuzzy data and compare it with other fuzzy process capability indices [J]. Expert Systems with Applications, 2011, 38 (6):6452-6457.

[8] Kurniati N, Yeh R H, Wu C W. A sampling scheme for resubmitted lots based on one-sided capability indices $[\mathrm{J}]$. Quality Technology \& Quantitative Management, 2015, 12 (4):501-515.

[9] Seifi S, Nezhad M S F. Variable sampling plan for resubmitted lots based on process capability index and Bayesian approach $[\mathrm{J}]$. The International Journal of Advanced Manufacturing Technology, 2017, 88 (9-12): 2547-2555.

[10] Montgomery D C. Introduction to Statistical Quality Control, New York: John Wiley \& Sons, 1985.

[11] Lee W C, Hong C W, Wu J W. Computational procedure of performance assessment of lifetime index of normal products with fuzzy data under the type II right censored sampling plan [J]. Journal of Intelligent \& Fuzzy Systems, 2015, 28 (4):1755-1773.

[12] Liu M. F., Ren H. P. Bayesian test procedure of lifetime performance index for exponential distribution under progressive type-II censoring. International Journal of Applied Mathematics \& Statistics, 2013, 32 (2):27-38.

[13] Wu C W, Shu M H, Chang Y N. Variable-sampling plans based on lifetime-performance index under exponential distribution with censoring and its extensions [J]. Applied Mathematical Modelling, 2018, 55:81-93.

[14] Soliman A E. Assessing the lifetime performance index using exponentiated frechet distribution with the progressive firstfailure-censoring scheme [J]. American Journal of Theoretical and Applied Statistics, 2014, 3 (6):167-176.

[15] Li L. Bayesian test for lifetime performance index of Ailamujia distribution under squared error loss function [J]. Pure and Applied Mathematics Journal, 2016, 5 (6):181-185.

[16] Zhao S, Song Y, Song L, et al. Estimation of ordered means of two sample exponential distributions under symmetric entropy loss [J]. Journal of Jilin University, 2007, 45 (1):44-48.

[17] Li L. Bayes estimation of Topp-Leone distribution under symmetric entropy loss function based on lower record values [J]. 2016, 4 (6):284.

[18] Lawless J F. Statistical Model and Methods for Lifetime Data. John Wiley \& Sons, New York, 1982.

[19] Balakrishnan N, Lin C T, and Chan P S. A comparison of two simple prediction intervals for exponential distribution. IEEE Transactions on Reliability, 2005, 54: 27-33.

[20] Gail M H and Gastwirth J L. A scale-free goodness of fit test for the exponential distribution based on the Gini statistics, Journal of the Royal Statistical Society, B, 1978, 40: 350-357. 